PROCEEDINGS OF THE

AMERICAN MATHEMATICAL SOCIETY

Volume 131, Number 8, Pages 2573-2576

S 0002-9939(03)06878-3

Article electronically published on February 26, 2003

\title{
COMPACT EINSTEIN WARPED PRODUCT SPACES WITH NONPOSITIVE SCALAR CURVATURE
}

\author{
DONG-SOO KIM AND YOUNG HO KIM \\ (Communicated by Wolfgang Ziller) \\ Dedicated to Professor Bang-yen Chen on the occasion of his sixtieth birthday

\begin{abstract}
We study Einstein warped product spaces. As a result, we prove the following: if $M$ is an Einstein warped product space with nonpositive scalar
\end{abstract} \\ curvature and compact base, then $M$ is simply a Riemannian product space.
}

\section{INTRODUCTION}

Let $B=\left(B^{m}, g_{B}\right)$ and $F=\left(F^{k}, g_{F}\right)$ be two Riemannian manifolds. We denote by $\pi$ and $\sigma$ the projections of $B \times F$ onto $B$ and $F$, respectively. For a positive smooth function $f$ on $B$ the warped product $M=B \times{ }_{f} F$ is the product $M=B \times F$ furnished with the metric tensor $g$ defined by $g=\pi^{*} g_{B}+f^{2} \sigma^{*} g_{F}$, where ${ }^{*}$ denotes the pull back. The function $f$ is referred to as the warping function. The notion of warped product $B \times{ }_{f} F$ generalizes that of a surface of revolution. It was introduced in 3 for studying manifolds of negative curvature.

A Riemannian manifold $M$ is called Einstein if its Ricci tensor Ric is proportional to the metric $g$, that is, Ric $=\lambda g$, where $\lambda$ is a constant on $M$. Obviously the Riemannian product $M=B \times F$ is Einstein if $B$ and $F$ are Einstein with the same scalar curvature. A warped product $B \times{ }_{f} F$ with a constant warping function $f$ can be considered as a Riemannian product.

In search of a new compact Einstein space in 2 (p. 265), A. L. Besse asked the following:

"Does there exist a compact Einstein warped product with nonconstant warping function?"

In this article, we give a negative partial answer as follows (cf. [1]):

Theorem 1. Let $M=B \times_{f} F$ be an Einstein warped product space with base $B$ a compact space. If $M$ has nonpositive scalar curvature, then the warped product is simply a Riemannian product.

Received by the editors August 14, 2000 and, in revised form, July 10, 2001.

2000 Mathematics Subject Classification. Primary 53B20, 53C20.

Key words and phrases. Einstein space, warped product, Ricci tensor, Hessian tensor, Ricci identity.

This work was supported by the Brain Korea 21 .

(C)2003 American Mathematical Society 


\section{Proofs}

We denote by $\operatorname{Ric}^{B}, \operatorname{Ric}^{F}$ the lifts to $M$ of the Ricci curvatures of $B$ and $F$, respectively. Then we have the following ([6] $)$ :

Proposition 2. The Ricci curvature Ric of the warped product $M=B \times_{f}$ F with $k=\operatorname{dim} F$ satisfies

(1) $\operatorname{Ric}(X, Y)=\operatorname{Ric}^{B}(X, Y)-\frac{k}{f} H^{f}(X, Y)$,

(2) $\operatorname{Ric}(X, V)=0$,

(3) $\operatorname{Ric}(V, W)=\operatorname{Ric}^{F}(V, W)-g(V, W) f^{\#}, f^{\#}=\frac{-\Delta f}{f}+\frac{k-1}{f^{2}} g_{B}(\nabla f, \nabla f)$ for any horizontal vectors $X, Y$ and any vertical vectors $V, W$, where $H^{f}$ and $\Delta f$ denote the Hessian of $f$ and the Laplacian of $f$ given by $-\operatorname{tr}\left(H^{f}\right)$, respectively.

Hence the Einstein equations become

Corollary 3. The warped product $M=B \times_{f}$ Fis Einstein with Ric $=\lambda g$ if and only if

(1.1) $\operatorname{Ric}_{B}=\lambda g_{B}+\frac{k}{f} H^{f}$,

(1.2) $\left(F, g_{F}\right)$ is Einstein with $\operatorname{Ric}_{F}=\mu g_{F}$,

$(1.3)-f \Delta f+(k-1)|\nabla f|^{2}+\lambda f^{2}=\mu$.

Now we prove a lemma.

Lemma 4. Let $f$ be a smooth function on a Riemannian manifold $B$. Then for any vector $X$, the divergence of the Hessian tensor $H^{f}$ satisfies

$$
\operatorname{div}\left(H^{f}\right)(X)=\operatorname{Ric}(\nabla f, X)-\Delta(d f)(X),
$$

where $\Delta=d \delta+\delta d$ denotes the Laplacian on $B$ acting on differential forms.

Proof. The well-known Ricci identity implies (cf. [5], p. 159)

$$
D^{2} d f(X, Y, Z)-D^{2} d f(Y, X, Z)=d f\left(R_{X Y} Z\right)
$$

for all vector fields $X, Y$, and $Z$ where $D_{X Y}^{2}=D_{X} D_{Y}-D_{D_{X} Y}$ denotes the second order covariant differential operator and $R_{X Y}=-D_{X} D_{Y}+D_{Y} D_{X}+D_{[X, Y]}$ is the curvature tensor acting on tensors as a derivation. Since $d f$ is closed, it is easily proved that

$$
D^{2} d f(X, Y, Z)=D^{2} d f(X, Z, Y)
$$

for any vector fields $X, Y$ and $Z$.

For a fixed $p \in B$ we may choose a local orthonormal frame $E_{1}, E_{2}, \cdots, E_{m}$ of the space $B$ such that $D_{E_{i}} E_{j}(p)=0$ for all $i, j$. Also, we may assume $D_{E i} Y(p)=0$ for a vector field $Y$. Taking the trace with respect to $X$ and $Z$ in (1.5) and using (1.6), we have

$$
\sum_{i}\left(D^{2} d f\right)\left(E_{i}, E_{i}, Y\right)=-d \Delta f(Y)+\operatorname{Ric}(Y, \nabla f)
$$

at $p$. Since $\operatorname{div} H^{f}(Y)=\sum_{i}\left(D^{2} d f\right)\left(E_{i}, E_{i}, Y\right)$ is straightforward, (1.4) is proved.

Proposition 5. Let $\left(B^{m}, g_{B}\right)$ be a compact Riemannian manifold of dimension $m \geqq 2$. Suppose that $f$ is a nonconstant smooth function on $B$ satisfying (1.1) for a constant $\lambda \in R$ and a natural number $k \in N$. Then $f$ satisfies (1.3) for a constant $\mu \in R$. Hence for a compact Einstein space $\left(F, g_{F}\right)$ of dimension $k$ with $\operatorname{Ric}_{F}=$ 
$\mu g_{F}$, we can make a compact Einstein warped product space $M=B \times_{f}$ F with $\operatorname{Ric}=\lambda g$.

Proof. By taking the trace of both sides of (1.1), we have

$$
S=m \lambda-\frac{k}{f} \Delta f
$$

where $S$ denotes scalar curvature of $B$ given by $\operatorname{tr}($ Ric). Note that the second Bianchi identity implies ([6], p. 88)

$$
d S=2 \operatorname{div}(\text { Ric })
$$

From (1.7) and (1.8), we obtain

$$
\operatorname{div} \operatorname{Ric}(X)=\frac{k}{2 f^{2}}\{\Delta f d f-f d(\Delta f)\}(X) .
$$

On the other hand, by definition we have

$$
\operatorname{div}\left(\frac{1}{f} H^{f}\right)(X)=\sum_{i}\left(D_{E i}\left(\frac{1}{f} H^{f}\right)\right)\left(E_{i}, X\right)=-\frac{1}{f^{2}} H^{f}(\nabla f, X)+\frac{1}{f} \operatorname{div} H^{f}(X)
$$

for any vector field $X$ and an orthonormal frame $E_{1}, E_{2}, \cdots, E_{m}$ of $B$. Since $H^{f}(X, \nabla f)=\left(D_{X} d f\right)(\nabla f)=\frac{1}{2} d\left(|\nabla f|^{2}\right)(X)$, the last equation becomes

$$
\operatorname{div}\left(\frac{1}{f} H^{f}\right)(X)=-\frac{1}{2 f^{2}} d\left(|\nabla f|^{2}\right)(X)+\frac{1}{f} \operatorname{div} H^{f}(X)
$$

for a vector field $X$ on $B$. Hence, from (1.1) and (1.4) it follows that

$$
\operatorname{div}\left(\frac{1}{f} H^{f}\right)=\frac{1}{2 f^{2}}\left\{(k-1) d\left(|\nabla f|^{2}\right)-2 f d(\Delta f)+2 \lambda f d f\right\} .
$$

But, (1.1) gives divRic $=\operatorname{div}\left(\frac{k}{f} H^{f}\right)$. Therefore, (1.9) and (1.10) imply that $d\left(-f \Delta f+(k-1)|\nabla f|^{2}+\lambda f^{2}\right)=0$, that is, $-f \Delta f+(k-1)|\nabla f|^{2}+\lambda f^{2}=\mu$ for some constant $\mu$. Thus the first part of the proposition is proved. For a compact Einstein manifold $\left(F, g_{F}\right)$ of dimension $k$ with $\operatorname{Ric}_{F}=\mu g_{F}$, we can construct a compact Einstein warped product $M=B \times{ }_{f} F$ by the sufficiencies of Corollary 3 .

Now we give the proof of Theorem 1. Note that (1.3) becomes

$$
\operatorname{div}(f \nabla f)+(k-2)|\nabla f|^{2}+\lambda f^{2}=\mu .
$$

By integrating (1.11) over $B$ we have

$$
\mu=\frac{k-2}{V(B)} \int_{B}|\nabla f|^{2}+\frac{\lambda}{V(B)} \int_{B} f^{2},
$$

where $V(B)$ denotes the volume of $B$.

1) Suppose $k \geq 3$. Let $p$ be a maximum point of $f$ on $B$. Then, we have $f(p)>0, \nabla f(p)=0$ and $\Delta f(p) \geq 0$. Hence from (1.3) and (1.12) we obtain the following:

$$
\begin{aligned}
0 & \leqq f(p) \Delta f(p) \\
& =\lambda f(p)^{2}-\mu \\
& =\frac{2-k}{V(B)} \int_{B}|\nabla f|^{2}+\frac{\lambda}{V(B)} \int_{B}\left(f(p)^{2}-f^{2}\right) \\
& \leqq 0
\end{aligned}
$$


The last inequality follows from the hypothesis on $\lambda$. Thus, $f$ is constant.

2) When $k=1,2$, we choose $q$ as a minimum point of $f$ on $B$. Then, we have $f(q)>0, \nabla f(q)=0$ and $\Delta f(q) \leq 0$. Hence we obtain from (1.3) and (1.12)

$$
\begin{aligned}
0 & \geqq f(q) \Delta f(q) \\
& =\lambda f(q)^{2}-\mu \\
& =\frac{2-k}{V(B)} \int_{B}|\nabla f|^{2}+\frac{\lambda}{V(B)} \int_{B}\left(f(q)^{2}-f^{2}\right) \\
& \geqq 0 .
\end{aligned}
$$

As in case 1), the last inequality follows from the hypothesis on $\lambda$. If $k=1$ or $\lambda<0$, then (1.13) shows that $f$ is constant. If $k=2$ and $\lambda=0,(1.11)$ and (1.12) imply that $f^{2}$ is harmonic on $B$, and hence $f$ is constant. This completes the proof of the theorem.

In a similar manner, we may prove the following (cf. [4]):

Remark 6. Let $(M, g)$ be a compact Riemannian manifold. If the Ricci tensor satisfies Ric $=\lambda g+H^{f}$ for a nonpositive constant $\lambda \in R$ and a smooth function $f$ on $M$, then $f$ is constant.

\section{ACKNOWLEDGEMENT}

The authors would like to express their deep thanks to the referee for valuable suggestions to improve the paper.

\section{REFERENCES}

1. J. K. Beem, P. E. Ehrlich and K. L. Easley, Global Lorentzian Geometry (2nd ed.), Marcel Dekker, Inc., New York (1996). MR 97f:53100

2. A. L. Besse, Einstein Manifolds, Springer-Verlag, Berlin-Heidelberg (1987). MR 88f:53087

3. R. L. Bishop and B. O'Neill, Manifolds of negative curvature, Trans. Amer. Math. Soc. 145 (1969), 1-49. MR 40:4891

4. D. DeTurck, Metrics with prescribed Ricci curvature, Seminar on Differential Geometry (S. T. Yau, ed.), Ann. of Math. Stud., vol. 102, Princeton Univ. Press, Princeton, NJ (1982), 525-537. MR 83e:53014

5. S. Gallot, D. Hulin and J. Lafontaine, Riemannian Geometry, Springer-Verlag, Berlin (1987). MR 88k:53001

6. B. O'Neill, Semi-Riemannian Geometry with applications to Relativity, Academic Press, New York (1983).

Department of Mathematics, College of Natural Sciences, Chonnam National UniVERSITY, KWANGJU, 500-757, KOREA

E-mail address: dosokim@chonnam.chonnam.ac.kr

Department of Mathematics, College of Natural Sciences, Kyungpook National UniVERSITY, TAEGU, 702-701, KorEA

E-mail address: yhkim@knu.ac.kr 